\title{
Factores de impacto de Biological Research: 1998-2004
}

\author{
Biological Research Impact Factors: 1998-2004
}

\author{
JAIME R. RAU
}

Laboratorio de Ecología, Departamento de Ciencias Básicas \& Programa IBAM, Universidad de Los Lagos, Osorno, Chile.

Entre las revistas científicas del área biológica experimental, Biological Research (Biol. Res.) es lejos la revista latinoamericana de corriente principal (Krauskopf \& Vera 1995) que tiene los factores de impacto (Amin \& Mabe 2000) e índices de inmediatez, sensu el Institute for Scientific Information (ISI), más altos de Latinoamérica (Science Citation Index, SCI-Journal Citation Reports, JCR 1999-2005; BR Sabe, 2001; Krauskopf, 2002; 2003). El SCI-JCR entrega estas estadísticas para el año previo (e.g., 2004, en este caso) y se publica a mediados del mes de junio de cada año. El SCI-JCR (2005) acaba de ser publicado y entrega los factores de impacto e índices de inmediatez calculados para el año 2004. El factor de impacto se calcula para cada año relacionando las citas del año de interés (numerador) respecto de los artículos publicados en los dos años previos (denominador). Los factores de impacto se ordenan por rango de valores ascendentes para cada una de las áreas disciplinarias en que el ISI clasifica las revistas que son indexadas en su base de datos. El índice de inmediatez es más exigente ya que se calcula solo para un horizonte de tiempo de un año (Krauskopf, 2003).

La tasa de publicación de manuscritos por Biol. Res. es de aproximadamente un $60 \%$ (Krauskopf, 2002), lo que supone una tasa de rechazo de ca. 40\%. Probablemente, altas exigencias en la aceptación y publicación de manuscritos pueden explicar que los factores de impacto e índices de inmediatez sean concomitantemente altos.

Entre las revistas científicas del área naturalista, la Revista Chilena de Historia Natural (Rev. Chil. Hist. Nat.) es la que tiene el factor de impacto más alto de Latinoamérica (Rau, 1997), el que para el año 2004 fue igual a 0,583 , y también tiene una tasa de rechazo de manuscritos de alrededor un 40\% (Jaksic, 1997; Rau, 1997). Ambas revistas son editadas por la Sociedad de Biología de Chile.

En este artículo analizaré la evolución temporal de los factores de impacto de Biol. Res., los cuantificaré usando un par de fórmulas de uso corriente en ecología (Rau, 1997), los ajustaré a un modelo matemático simple y en base a lo anterior realizaré una predicción sobre su factor de impacto futuro.

Biol. Res. aparece por primera vez en las listas del SCI-JCR en 1998 (publicadas en 1999). Al momento de escribir este trabajo, el SCI-JCR (2005) más reciente entrega información actualizada sobre su factor de impacto e índice de inmediatez para el año 2004. Estos valores han sido, respectivamente, iguales a 2,173 y 0,757 . En cuanto a su índice de inmediatez, a nivel mundial Biol. Res. ha sido la tercera de 51 revistas biológicas incluidas en la base de datos del ISI (Krauskopf, 2003). En lo que se refiere a su factor de impacto, Biol. Res. ocupa actualmente el lugar 16 de 64 revistas ISI en el área disciplinaria correspondiente a la biología. Es decir, se halla en el percentil 25 superior de la 
distribución de valores para los factores de impacto.

En la Tabla 1 presento la evolución temporal del factor de impacto de Biol. Res. entre 1998-2004 ( $\mathrm{n}=7$ años). $\mathrm{Su}$ valor promedio ha sido igual a 1,160 , con un coeficiente de variación ( $\mathrm{CV}$ ) mayor que un $48 \%$. Su valor se ha incrementado marcadamente desde 0,867 (1998) a 2,173 (2004). Esto indica que el factor de impacto de esta revista ha aumentado 2,5 veces en esos siete años. El aumento ha sido exponencial y los datos de la Tabla 1 no difieren de una distribución exponencial (Prueba estadística de Kolmogorov-Smirnov para una muestra: $\mathrm{DN}=0,345 ; \mathrm{P}=0,377)$.

Utilizando la fórmula 2, descrita y explicada en Rau (1997), se obtiene [1n 2004 - ln 1998/7]) que el crecimiento (r) del factor de impacto de Biol. Res. hasta los últimos siete años, compilados por el SCIJCR (1999-2005), ha sido de un $r=0,131$ $(\ln 2,173$ - $\ln 0,867 / 7)$ o alrededor de un $13 \%$ anual. En la Figura 1 se muestra el buen ajuste (coeficiente de correlación producto-momento de Pearson $=0,912 \mathrm{y}$ error estándar $=0,252$ ) a una curva exponencial, obtenido con el programa computacional CurveExpert versión 1.3 (http: //www.curveexpert.webhop.biz/).

TABLA 1

Factores de impacto de Biological Research durante el período 1998-2004

\begin{tabular}{lc}
\hline Año & Factor de impacto \\
\hline 1998 & 0,867 \\
1999 & 0,490 \\
2000 & 0,680 \\
2001 & 1,154 \\
2002 & 1,444 \\
2003 & 1,310 \\
2004 & 2,173 \\
Promedio $[\mathrm{CV}(\%)]$ & $1,160(48,4 \%)$ \\
\hline
\end{tabular}

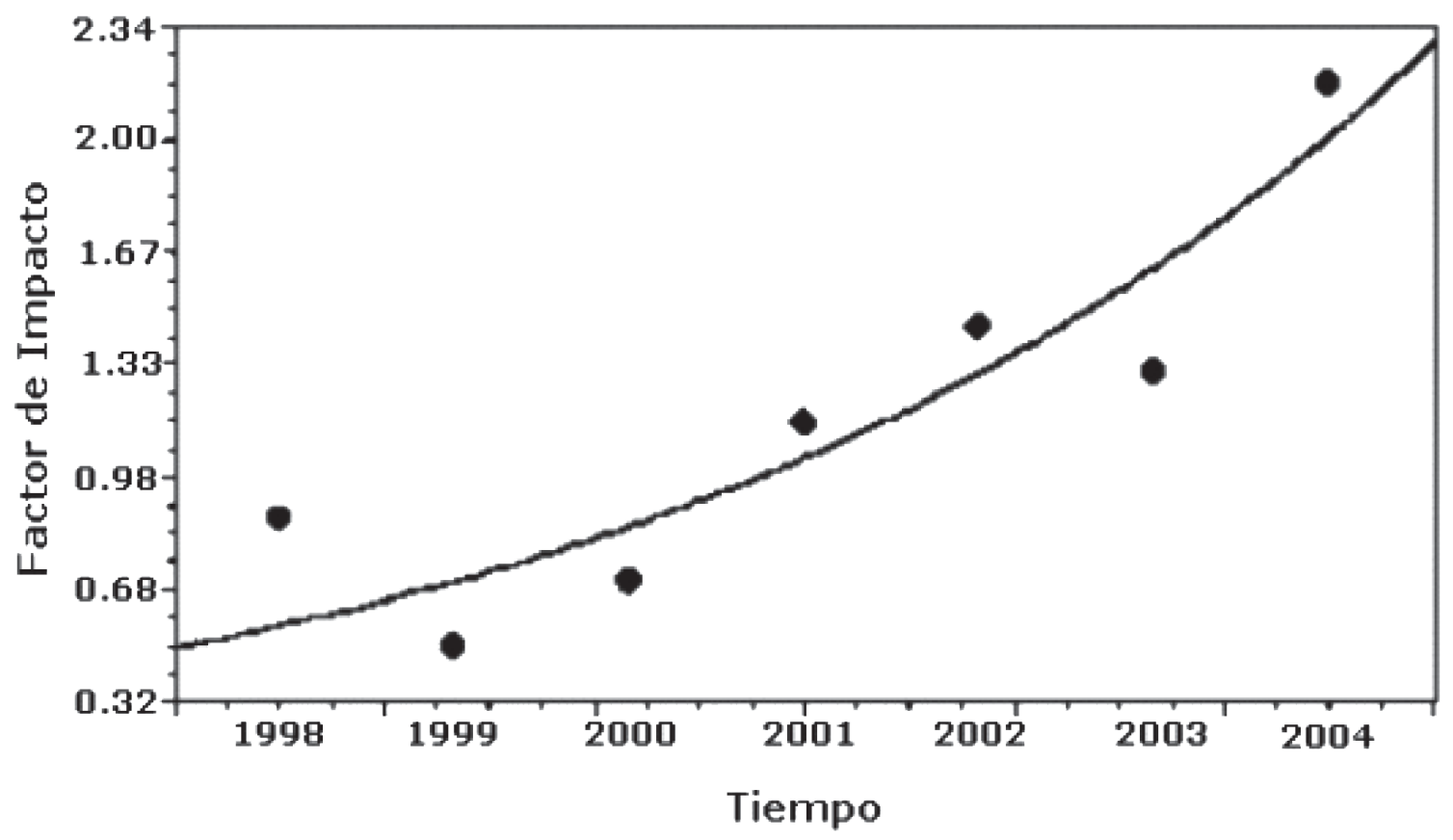

Figura 1: Evolución temporal de los factores de impacto de Biological Research durante el período 1998-2004 y su ajuste a un modelo exponencial con el programa computacional CurveExpert versión 1.3 . 
De acuerdo con la fórmula 3, también descrita y explicada en Rau (1997), para una serie de datos que sigue un modelo exponencial puede calcularse $(\ln 2 / r)$ que el actual factor de impacto (2004) de Biol. Res. $(2,173)$ se duplicará en solo un poco más de 5 años $(0,693 / 0,131)$. Si esta hipótesis se cumple, entonces puede predecirse que en el SCI-JCR del año 2010 (que entregará el factor de impacto de esta revista para el año 2009), el factor de impacto de Biol. Res. debería ser algo mayor que 4 (en realidad, 4,346). Haciendo un ejercicio similar, predije anteriormente que el factor de impacto teórico para la Rev. Chil. Hist. Nat. sería igual a 0,552 en 1998 (Rau, 1997). El factor de impacto empírico para el mismo año fue en realidad igual a 0,330 . Por lo tanto, para una serie de tiempo de solo cinco años maximicé $1,673(0,552 / 0,330)$ veces mi predicción. Si así también fuese para Biol. Res., el factor de impacto que ahora predigo para Biol. Res. podría solo ser igual a 2,598 $(4,346 / 1,673)$. Este valor es considerado de todas maneras como un alto índice de impacto $(>1,0)$, cuando se le usa para evaluar los curricula de investigadores y bonificar sus publicaciones en revistas ISI.

\section{AGRADECIMIENTOS}

El autor desea reconocer y destacar el muy oportuno y gentil aporte de información por parte de Pamela Blazick (muy especialmente), actual editora del JCR, Michael G. Moskow, ambos del ISI, EE.UU., y Marcela Aguirre del Conicyt, Chile. Finalmente, a Macarena Rau C. por su ayuda en la confección de la figura.

\section{REFERENCIAS}

AMIN M, MABE M (2000) Impact Factors: Use and abuse. Perspectives in Publishing 1: 1-6

BR SABE (2001) Immediacy index en la Categoría "Biology": Biological Research alcanza el quinto lugar entre sus congéneres de acuerdo al Journal of Citation Reports (2000). Biol Res 34: 20

JAKSIC FM (1997) The first hundred years of the Revista Chilena de Historia Natural. Revista Chilena de Historia Natural 70: 5-8

KRAUSKOPF M, VERA MI (1995) Las revistas latinoamericanas de corriente principal: Indicadores y estrategias para su consolidación. Interciencia 20: 144148

KRAUSKOPF M (2002) Biological Research Factor de Impacto 1,154. Biol Res 35: 331

KRAUSKOPF M (2003) La falta de un adecuado reconocimiento de las capacidades existentes afecta el desarrollo científico en Chile. Biol Res 36: 139-140

RAU JR (1997) Factores de impacto de la Revista Chilena de Historia Natural: 1991-1995. Revista Chilena de Historia Natural 70: 453-457

SCIENCE CITATION INDEX (1999-2004) Journal Citation Report. Institute for Scientific Information. Philadelphia, Pennsylvania, USA. 